\title{
On some distance-regular graphs with many vertices
}

\author{
Dean Crnković ${ }^{1} \cdot$ Sanja Rukavina ${ }^{1} \cdot$ Andrea Švob $^{1}$ \\ Received: 10 October 2018 / Accepted: 19 April 2019 / Published online: 18 May 2019 \\ (c) Springer Science+Business Media, LLC, part of Springer Nature 2019
}

\begin{abstract}
We construct distance-regular graphs, including strongly regular graphs, admitting a transitive action of the Chevalley groups $G_{2}(4)$ and $G_{2}(5)$, the orthogonal group $O(7,3)$ and the Tits group $T={ }^{2} F_{4}(2)^{\prime}$. Most of the constructed graphs have more than 1000 vertices, and the number of vertices goes up to 28431 . Some of the obtained graphs are new.
\end{abstract}

Keywords Strongly regular graph · Distance-regular graph · Orthogonal group ·

Chevalley group · Tits group

Mathematics Subject Classification 05E30 - 05E18

\section{Introduction}

A construction of distance-regular graphs (DRGs), and especially strongly regular graphs (SRGs), from finite groups gave an important contribution to the graph theory and the design theory (see [4,6,19]). Recently, in [11] the authors found new SRGs admitting a transitive action of some finite simple groups. Research presented in this paper shows how one can use groups as a tool to produce wide range of regular graphs. Moreover, in that way an insight to particular groups is given and some DRGs (and especially SRGs) with many vertices are constructed. In the literature, and also implemented in Sagemath (see [8,23]), there are a few examples of SRGs and DRGs with many vertices that do not belong to some infinite family of graphs. The expression "many vertices" in this paper refers to the number of vertices that is out of range given

$凶$ Andrea Švob

asvob@math.uniri.hr

Dean Crnković

deanc@math.uniri.hr

Sanja Rukavina

sanjar@math.uniri.hr

1 Department of Mathematics, University of Rijeka, Radmile Matejčić 2, 51000 Rijeka, Croatia 
in $[5,6]$. For the sake of completeness of the presented results, we also include obtained graphs on less than 1000 vertices.

We assume that the reader is familiar with the basic facts of the group theory, the theory of strongly regular graphs and the theory of distance-regular graphs. We refer the reader to $[9,20]$ for relevant background reading in the group theory, to $[4,25]$ for the theory of strongly regular graphs, and to [6,13] for the theory of distance-regular graphs.

In this paper we study the exceptional groups of Lie type $G_{2}(4)$ and $G_{2}(5)$, the orthogonal group $O(7,3)$ and the Tits group $T$, which are the simple groups of orders $251596800,5859000000,4585351680$ and 17971200 , respectively. We refer the reader to $[9,28]$ for more details about these groups.

Using the method outlined in Sect. 3, we constructed and classified SRGs and DRGs of diameter $d \geq 3$ from above-mentioned simple groups as follows:

- up to 20000 vertices admitting a primitive action of the group $G_{2}(4)$, and up to 13000 vertices admitting an imprimitive action of the group $G_{2}(4)$,

- up to 400000 vertices admitting a primitive action of the group $G_{2}(5)$, and up to 15000 vertices admitting an imprimitive action of the group $G_{2}(5)$,

- up to 250000 vertices admitting a primitive action of the group $O(7,3)$, and up to 10000 vertices admitting an imprimitive action of the group $O(7,3)$,

- up to 12000 vertices admitting a primitive action of the group $T$, and up to 7000 vertices admitting an imprimitive action of the group $T$.

Based on $[4,5,17]$ and the other literature, we conclude that the constructed strongly regular graphs with parameters $(28431,3150,621,315)$ and $(28431,2880,324,288)$ are the first known examples of SRGs with these parameters.

Note that in the paper we use the notation from [9] to describe the structures of the groups. To find the graphs and compute their full automorphism groups, we used programmes written for Magma [3] and GAP [15]. The constructed SRGs and DRGs can be found at the link:

http://www.math.uniri.hr/ asvob/DRGs_manyVertices.zip.

\section{Preliminaries}

In this section we define coherent configurations and association schemes, which are the tools for the construction of graphs presented in this paper. We also give basic definitions and properties of DRGs and SRGs.

Definition 1 A coherent configuration on a finite non-empty set $\Omega$ is an ordered pair $(\Omega, \mathcal{R})$ with $\mathcal{R}=\left\{R_{0}, R_{1}, \ldots, R_{d}\right\}$ a set of non-empty relations on $\Omega$, such that the following axioms hold.

(i) $\sum_{i=0}^{t} R_{i}$ is the identity relation, where $\left\{R_{0}, R_{1}, \ldots, R_{t}\right\} \subseteq\left\{R_{0}, R_{1}, \ldots, R_{d}\right\}$.

(ii) $\mathcal{R}$ is a partition of $\Omega^{2}$.

(iii) For every relation $R_{i} \in \mathcal{R}$, its converse $R_{i}^{T}=\left\{(y, x):(x, y) \in R_{i}\right\}$ is in $\mathcal{R}$. 
(iv) There are constants $p_{i j}^{k}$ known as the intersection numbers of the coherent configuration $\mathcal{R}$, such that for $(x, y) \in R_{k}$, the number of elements $z$ in $\Omega$ for which $(x, z) \in R_{i}$ and $(z, y) \in R_{j}$ equals $p_{i j}^{k}$.

We say that a coherent configuration is homogeneous if it contains the identity relation, i.e., if $R_{0}=I$. If $\mathcal{R}$ is a set of symmetric relations on $\Omega$, then a coherent configuration is called symmetric. A symmetric coherent configuration is homogeneous (see [7]). Symmetric coherent configurations are introduced by Bose and Shimamoto in [2] and called association schemes. An association scheme with relations $\left\{R_{0}, R_{1}, \ldots, R_{d}\right\}$ is called a $d$-class association scheme.

Let $\Gamma$ be a graph with diameter $d$, and let $\delta(u, v)$ denote the distance between vertices $u$ and $v$ of $\Gamma$. The $i$ th-neighborhood of a vertex $v$ is the set $\Gamma_{i}(v)=\{w$ : $\delta(v, w)=i\}$. Similarly, we define $\Gamma_{i}$ to be the $i$ th-distance graph of $\Gamma$, that is, the vertex set of $\Gamma_{i}$ is the same as for $\Gamma$, with adjacency in $\Gamma_{i}$ defined by the $i$ th-distance relation in $\Gamma$. We say that $\Gamma$ is distance-regular if the distance relations of $\Gamma$ give the relations of a $d$-class association scheme, that is, for every choice of $0 \leq i, j, k \leq d$, all vertices $v$ and $w$ with $\delta(v, w)=k$ satisfy $\left|\Gamma_{i}(v) \cap \Gamma_{j}(w)\right|=p_{i j}^{k}$ for some constant $p_{i j}^{k}$. In a distance-regular graph, we have that $p_{i j}^{k}=0$ whenever $i+j<k$ or $k<|i-j|$. A distance-regular graph $\Gamma$ is necessarily regular with degree $p_{11}^{0}$; more generally, each distance graph $\Gamma_{i}$ is regular with degree $k_{i}=p_{i i}^{0}$.

An equivalent definition of distance-regular graphs is the existence of the constants $b_{i}=p_{i+1,1}^{i}$ and $c_{i}=p_{i-1,1}^{i}$ for $0 \leq i \leq d$ (notice that $b_{d}=c_{0}=0$ ). The sequence $\left\{b_{0}, b_{1}, \ldots, b_{d-1} ; c_{1}, c_{2}, \ldots, c_{d}\right\}$, where $d$ is the diameter of $\Gamma$ is called the intersection array of $\Gamma$. Clearly, $b_{0}=k, b_{d}=c_{0}=0, c_{1}=0$.

A regular graph is strongly regular with parameters $(v, k, \lambda, \mu)$ if it has $v$ vertices, degree $k$, and if any two adjacent vertices are together adjacent to $\lambda$ vertices, while any two non-adjacent vertices are together adjacent to $\mu$ vertices. A strongly regular graph with parameters $(v, k, \lambda, \mu)$ is usually denoted by $\operatorname{SRG}(v, k, \lambda, \mu)$. A strongly regular graph is a distance-regular graph with diameter 2 whenever $\mu \neq 0$. The intersection array of an SRG is given by $\{k, k-1-\lambda ; 1, \mu\}$.

\section{SRGs and DRGs constructed from the groups}

Let $G$ be a finite permutation group acting on the finite set $\Omega$. This action induces the action of the group $G$ on the set $\Omega \times \Omega$. For more information see [27]. The orbits of this action are the sets of the form $\{(\alpha g, \beta g): g \in G\}$. If $G$ is transitive, then $\{(\alpha, \alpha): \alpha \in \Omega\}$ is one such orbit. If the rank of $G$ is $r$, then it has $r$ orbits on $\Omega \times \Omega$. Let $|\Omega|=n$ and $\Delta_{i}$ is one of these orbits. We say that the $n \times n$ matrix $A_{i}$, with rows and columns indexed by $\Omega$ and entries

$$
A_{i}(\alpha, \beta)=\left\{\begin{array}{l}
1, \text { if }(\alpha, \beta) \in \Delta_{i} \\
0, \text { otherwise. }
\end{array}\right.
$$

is called the adjacency matrix for the orbit $\Delta_{i}$. 
Let $A_{0}, \ldots, A_{r-1}$ be the adjacency matrices for the orbits of $G$ on $\Omega \times \Omega$. These satisfy the following conditions.

(i) $A_{0}=I$, if $G$ is transitive on $\Omega$. If $G$ has $s$ orbits on $\Omega$, then $I$ is a sum of $s$ adjacency matrices.

(ii) $\sum_{i} A_{i}=J$, where $J$ is the all-one matrix.

(iii) If $A_{i}$ is an adjacency matrix, then so is its transpose $A_{i}^{T}$.

(iv) If $A_{i}$ and $A_{j}$ are adjacency matrices, then their product is an integer-linearcombination of adjacency matrices.

If $A_{i}$ is symmetric, then the corresponding orbit is called self-paired. Further, if $A_{i}=A_{j}^{T}$, then the corresponding orbits are called mutually paired.

The graphs obtained in this paper are constructed using the method described in [10] which can be rewritten in terms of coherent configurations in the following way.

Theorem 1 Let $G$ be a finite permutation group acting transitively on the set $\Omega$ and $A_{0}, \ldots, A_{d}$ be the adjacency matrices for orbits of $G$ on $\Omega \times \Omega$. Let $\left\{B_{1}, \ldots, B_{t}\right\} \subseteq$ $\left\{A_{1}, \ldots, A_{d}\right\}$ be a set of adjacency matrices for the self-paired or mutually paired orbits. Then, $M=\sum_{i=1}^{t} B_{i}$ is the adjacency matrix of a regular graph $\Gamma$. The group $G$ acts transitively on the set of vertices of the graph $\Gamma$.

Using this method one can construct all regular graphs admitting a transitive action of the group $G$. We will be interested only in those regular graphs that are distanceregular, and especially strongly regular.

The running time complexity of the algorithm used for the construction of the graphs depends on a number of parameters, such as the size of the used group and subgroup, the number of orbits of the stabilizer and the number of self-paired and mutually paired orbits in a particular case. For example, one of the cases that we considered but were not able to solve is the following. The group $O(7,3)$ has the subgroup of order 113824 and index 331695. The number of orbits of the stabilizer is 102 which makes the computation impossible to finish in a reasonable time. Moreover, working with a structure defined on 331695 elements is difficult because of the used memory space.

\subsection{SRGs and DRGs from the group $G_{2}(4)$}

The group $G_{2}(4)$ is the simple group of order $251596800=2^{12} \cdot 3^{3} \cdot 5^{2} \cdot 7 \cdot 13$. Up to conjugation it has 4300 subgroups, 8 of which are maximal. It belongs to Chevalley's exceptional groups of Lie type. The group $G_{2}(4)$ has 9 conjugacy classes of subgroups up to the index 13000. In Table 1 we give the list of all the subgroups $H_{i}^{1} \leq G_{2}(4)$ which lead to the construction of SRGs or DRGs of diameter $d \geq 3$.

Using the method described in Theorem 1, we obtained all DRGs with at most 20000 vertices on which the group $G_{2}(4)$ acts primitively, and all DRGs with at most 13000 vertices on which the group $G_{2}(4)$ acts imprimitively (transitively but not primitively).

Theorem 2 Up to isomorphism there are exactly five strongly regular graphs and exactly two distance-regular graphs of diameter $d \geq 3$ with at most 20000 vertices, admitting a primitive action of the group $G_{2}(4)$, and exactly one strongly regular graph and exactly one distance-regular graph of diameter $d \geq 3$ with at 
most 13000 vertices, admitting an imprimitive action of the group $G_{2}(4)$. The SRGs have parameters $(416,100,36,20),(1365,340,83,85),(2080,1008,480,496)$, (2016, 975, 462, 480) and (4095, 2046, 1021, 1023), and the DRGs have 1365 and 6825 vertices, respectively. Details about the obtained strongly regular graphs are given in Table 2, and details about the obtained DRGs with $d \geq 3$ are given in Table 3.

Remark 1 The SRGs $\Gamma_{2}^{1}$ and $\Gamma_{3}^{1}$ are connected with the generalized hexagon $G H(4,4)$ of order $(4,4)$ [or shortly $H(4)$ ]. It is well known that the distance 3 graph of the point graph of a generalized hexagon $G H(4,4)$ is strongly regular. Moreover, according to [26, Corollary 3.5.7] the generalized hexagon $H(q)$ is self-dual if and only if $q=3^{h}$. Therefore, the generalized hexagon $H(4)$ is not self-dual. The graph $\Gamma_{3}^{1}$ is the distance 3 graph of the point graph of the generalized hexagon $H(4)$, and the strongly regular graph $\Gamma_{2}^{1}$ is the distance 3 graph of the point graph of the dual of the generalized

Table 1 Subgroups of the group $G_{2}(4)$

\begin{tabular}{llcccc}
\hline Subgroup & Structure & Order & Index & Rank & Primitive \\
\hline$H_{1}^{1}$ & $J_{2}$ & 604800 & 416 & 3 & Yes \\
$H_{2}^{1}$ & $2^{2+8}:\left(3 \times A_{5}\right)$ & 184320 & 1365 & 4 & Yes \\
$H_{3}^{1}$ & $2^{4+6}:\left(A_{5} \times 3\right)$ & 184320 & 1365 & 4 & Yes \\
$H_{4}^{1}$ & $U(3,4): Z_{2}$ & 124800 & 2016 & 3 & Yes \\
$H_{5}^{1}$ & $3 \cdot L_{3}(4): 2_{3}$ & 120960 & 2080 & 4 & Yes \\
$H_{6}^{1}$ & $2^{6}:\left(2^{4}: A_{5}\right)$ & 61440 & 4095 & 8 & No \\
$H_{7}^{1}$ & $\left(2^{2} \times\left(\left(2^{4}: 2\right): 2\right)\right):\left(A_{4} \times A_{4}\right)$ & 36864 & 6825 & 12 & No \\
\hline
\end{tabular}

Table 2 SRGs constructed from the group $G_{2}(4)$

\begin{tabular}{lll}
\hline Graph $\Gamma$ & Parameters & Aut $(\Gamma)$ \\
\hline$\Gamma_{1}^{1}=\Gamma\left(G_{2}(4), H_{1}^{1}\right)$ & $(416,100,36,20)$ & $G_{2}(4): Z_{2}$ \\
$\Gamma_{2}^{1}=\Gamma\left(G_{2}(4), H_{2}^{1}\right)$ & $(1365,340,83,85)$ & $G_{2}(4): Z_{2}$ \\
$\Gamma_{3}^{1}=\Gamma\left(G_{2}(4), H_{3}^{1}\right)$ & $(1365,340,83,85)$ & $O(7,4): Z_{2}$ \\
$\Gamma_{4}^{1}=\Gamma\left(G_{2}(4), H_{4}^{1}\right)$ & $(2016,975,462,480)$ & $O(7,4): Z_{2}$ \\
$\Gamma_{5}^{1}=\Gamma\left(G_{2}(4), H_{5}^{1}\right)$ & $(2080,1008,480,496)$ & $O(7,4): Z_{2}$ \\
$\Gamma_{6}^{1}=\Gamma\left(G_{2}(4), H_{6}^{1}\right)$ & $(4095,2046,1021,1023)$ & $O(13,2)$ \\
\hline
\end{tabular}

Table 3 DRGs constructed from the group $G_{2}(4), d \geq 3$

\begin{tabular}{lllll}
\hline Graph $\Gamma$ & Number of vertices & Diameter & Intersection array & Aut $(\Gamma)$ \\
\hline$\Gamma_{7}^{1}=\Gamma\left(G_{2}(4), H_{2}^{1}\right)$ & 1365 & 3 & $\{20,16,16 ; 1,1,5\}$ & $G_{2}(4): Z_{2}$ \\
$\Gamma_{8}^{1}=\Gamma\left(G_{2}(4), H_{3}^{1}\right)$ & 1365 & 3 & $\{20,16,16 ; 1,1,5\}$ & $G_{2}(4): Z_{2}$ \\
$\Gamma_{9}^{1}=\Gamma\left(G_{2}(4), H_{7}^{1}\right)$ & 6825 & 6 & $\{8,4,4,4,4,4 ; 1,1,1,1,1,2\}$ & $G_{2}(4): Z_{2}$ \\
\hline
\end{tabular}


hexagon $H(4)$. For more information see [14]. The graphs $\Gamma_{3}^{1}$ and $\Gamma_{6}^{1}$ are $O(7,4)$ and $O(13,2)$ graphs, respectively (see [5]). The graphs $\Gamma_{1}^{1}, \Gamma_{3}^{1}, \Gamma_{4}^{1}, \Gamma_{5}^{1}$ and $\Gamma_{6}^{1}$ are rank 3 graphs. Strongly regular graph $\Gamma_{1}^{1}$ is known as $G_{2}(4)$ graph. It is locally the Janko graph (see [18]), and the second subconstituent of the Suzuki tower.

Remark 2 SRGs denoted by $\Gamma_{2}^{1}, \Gamma_{3}^{1}$ and $\Gamma_{6}^{1}$ can be constructed from symmetric incidence matrices with all-one diagonal of symmetric block designs with parameters $(1365,341,85)$ and $(4095,2047,1023)$. The full automorphism groups of the designs and the corresponding SRGs do not have to be the same. In Table 4 we give the details about the obtained designs.

Remark 3 The graphs $\Gamma_{7}^{1}$ and $\Gamma_{8}^{1}$ belong to the family of graphs of Lie type from Chevalley groups (see [6]). The graph $\Gamma_{9}^{1}$ is the generalized dodecagon of order $(4,1)$ (see [12]).

\subsection{SRGs and DRGs from the group $G_{2}(5)$}

The group $G_{2}(5)$ is the simple group of order $5859000000=2^{6} \cdot 3^{5} \cdot 5^{6} \cdot 7 \cdot 31$. Up to conjugation it has 824 subgroups, 7 of which are maximal. It belongs to Chevalley's exceptional groups of Lie type. The group $G_{2}(5)$ has 7 conjugacy classes of subgroups up to the index 15000. In Table 5 we give the list of all the subgroups $H_{i}^{2} \leq G_{2}(5)$ which lead to the construction of SRGs or DRGs of diameter $d \geq 3$.

Using the method described in Theorem 1, we obtained all DRGs with at most 400000 vertices on which the group $G_{2}(5)$ acts primitively, and all DRGs with at most 15000 vertices on which the group $G_{2}(5)$ acts imprimitively.

Theorem 3 Up to isomorphism there are exactly six strongly regular graphs and exactly two distance-regular graphs of diameter $d \geq 3$ with at most 400000 vertices, admitting a primitive action of the group $G_{2}(5)$. The SRGs have parameters $(3906,780,154,156),(7875,1550,325,300),(7875,3224,1348,1300)$, (7750,

Table 4 Symmetric block designs constructed from the group $G_{2}(4)$

\begin{tabular}{lll}
\hline Design $\mathcal{D}$ & Parameters & Aut $(\mathcal{D})$ \\
\hline $\mathcal{D}_{1}^{1}=\Gamma\left(G_{2}(4), H_{2}^{1}\right)$ & $2-(1365,341,58)$ & $G_{2}(4): Z_{2}$ \\
$\mathcal{D}_{2}^{1}=\Gamma\left(G_{2}(4), H_{3}^{1}\right)$ & $2-(1365,341,58)$ & $P S L(6,4): S_{3}$ \\
$\mathcal{D}_{3}^{1}=\Gamma\left(G_{2}(4), H_{6}^{1}\right)$ & $2-(4095,2047,1023)$ & $P S L(12,2)$ \\
\hline
\end{tabular}

Table 5 Subgroups of the group $G_{2}(5)$

\begin{tabular}{llllll}
\hline Subgroup & Structure & Order & Index & Rank & Primitive \\
\hline$H_{1}^{2}$ & $5^{1+4}: G L_{2}(5)$ & 1500000 & 3906 & 4 & Yes \\
$H_{2}^{2}$ & $5^{2+3}: G L_{2}(5)$ & 1500000 & 3906 & 4 & Yes \\
$H_{3}^{2}$ & $3 . U_{3}(5): 2$ & 756000 & 7750 & 4 & Yes \\
$H_{4}^{2}$ & $L_{3}(5): 2$ & 744000 & 7875 & 5 & Yes \\
\hline
\end{tabular}


3024, 1148, 1200) and (7750, 1575, 300, 325), and the DRGs have 3906 vertices. Details about the obtained SRGs are given in Table 6 and about the obtained DRGs with $d \geq 3$ are given in Table 7 .

Remark 4 The SRGs $\Gamma_{1}^{2}$ and $\Gamma_{2}^{2}$ are connected with the generalized hexagon $G H(5,5)$ of order $(5,5)$ [or shortly $H(5)]$. The graph $\Gamma_{2}^{2}$ is the distance 3 graph of the point graph of the generalized hexagon $H(5)$, and the strongly regular graph $\Gamma_{1}^{2}$ is the distance 3 graph of the point graph of the dual of the generalized hexagon $H(5)$. The graph $\Gamma_{2}^{2}$ is a rank 3 graph and known as the $O(7,5)$ graph. Further, strongly regular graphs $\Gamma_{1}^{2}$ and $\Gamma_{2}^{2}$ can be constructed from a symmetric incidence matrix with allone diagonal of a symmetric block design with parameters $2-(3906,781,156)$. The full automorphism groups of symmetric block designs in these cases are the same as the automorphism groups of the corresponding SRGs. The SRGs with parameters $(7875,1550,325,300),(7875,3224,1348,1300),(7750,3024,1148,1200)$ and $(7750,1575,300,325)$ belong to families of SRGs related to non-singular quadrics in $P G(2 m, q)$, described in [17, Chapters 7C,7D], for $m=3$ and $q=5$. We checked imprimitive representations of the group $G_{2}(5)$ up to the degree 15000 , and proved that there are no SRGs in these cases.

Remark 5 The distance-regular graphs $\Gamma_{7}^{2}$ and $\Gamma_{8}^{2}$ belong to the family of graphs of Lie type from Chevalley groups. See [6] for more information. We checked imprimitive representations of the group $G_{2}(5)$ up to the degree 15000 , and proved that there are no DRGs in these cases.

Table 6 SRGs constructed from the group $G_{2}(5)$

\begin{tabular}{lll}
\hline Graph $\Gamma$ & Parameters & Aut $(\Gamma)$ \\
\hline$\Gamma_{1}^{2}=\Gamma\left(G_{2}(5), H_{1}^{2}\right)$ & $(3906,780,154,156)$ & $G_{2}(5)$ \\
$\Gamma_{2}^{2}=\Gamma\left(G_{2}(5), H_{2}^{2}\right)$ & $(3906,780,154,156)$ & $O(7,5): Z_{2}$ \\
$\Gamma_{3}^{2}=\Gamma\left(G_{2}(5), H_{3}^{2}\right)$ & $(7750,1575,300,325)$ & $O(7,5)$ \\
$\Gamma_{4}^{2}=\Gamma\left(G_{2}(5), H_{3}^{2}\right)$ & $(7750,3024,1148,1200)$ & $O(7,5)$ \\
$\Gamma_{5}^{2}=\Gamma\left(G_{2}(5), H_{4}^{2}\right)$ & $(7875,3224,1348,1300)$ & $O(7,5)$ \\
$\Gamma_{6}^{2}=\Gamma\left(G_{2}(5), H_{4}^{2}\right)$ & $(7875,1550,325,300)$ & $O(7,5)$ \\
\hline
\end{tabular}

Table 7 DRGs constructed from the group $G_{2}(5), d \geq 3$

\begin{tabular}{lllll}
\hline Graph $\Gamma$ & Number of vertices & Diameter & Intersection array & $\operatorname{Aut}(\Gamma)$ \\
\hline$\Gamma_{7}^{2}=\Gamma\left(G_{2}(5), H_{1}^{2}\right)$ & 3906 & 3 & $\{30,25,25 ; 1,1,6\}$ & $G_{2}(5)$ \\
$\Gamma_{8}^{2}=\Gamma\left(G_{2}(5), H_{2}^{2}\right)$ & 3906 & 3 & $\{30,25,25 ; 1,1,6\}$ & $G_{2}(5)$ \\
\hline
\end{tabular}


Table 8 Subgroups of the group $O(7,3)$

\begin{tabular}{llccll}
\hline Subgroup & Structure & Order & Index & Rank & Primitive \\
\hline$H_{1}^{3}$ & $2 U_{4}(3): 2$ & 13063680 & 351 & 3 & Yes \\
$H_{2}^{3}$ & $3^{5}: U_{4}(2): 2$ & 12597120 & 364 & 3 & Yes \\
$H_{3}^{3}$ & $L_{4}(3): 2$ & 12130560 & 378 & 3 & Yes \\
$H_{4}^{3}$ & $G_{2}(3)$ & 4245696 & 1080 & 3 & Yes \\
$H_{5}^{3}$ & $3^{3+3}: L_{3}(3)$ & 4094064 & 1120 & 4 & Yes \\
$H_{6}^{3}$ & $2^{6}: A_{7}$ & 161280 & 28431 & 12 & Yes \\
\hline
\end{tabular}

\subsection{SRGs and DRGs from the group $0(7,3)$}

The group $O(7,3)$ is the simple group of order $4585351680=2^{9} \cdot 3^{9} \cdot 5 \cdot 7 \cdot 13$. Up to conjugation it has 7735 subgroups, 15 of which are maximal. The group $O(7,3)$ has 15 conjugacy classes of subgroups up to the index 10000. In Table 8 we give the list of all the subgroups $H_{i}^{3} \leq O(7,3)$ which lead to the construction of SRGs or DRGs of diameter $d \geq 3$.

Using the method described in Theorem 1, we obtained all DRGs with at most 250000 vertices on which the group $O(7,3)$ acts primitively, and all DRGs with at most 10000 vertices on which the group $O(7,3)$ acts imprimitively.

Theorem $4 U$ to to isomorphism there are exactly seven SRGs and exactly one DRG of diameter $d \geq 3$ with at most 250000 vertices, admitting a primitive action of the orthogonal group $O(7,3)$. The SRGs have parameters $(351,126,45,45)$, (364, 120, 38, 40), (378, 117, 36, 36), (1080, 351, 126, 108), (1120, 390, 146, 130), $(28431,2880,324,288)$ and $(28431,3150,621,315)$, and the DRG with $d \geq 3$ has 1120 vertices. Details about the obtained SRGs are given in Table 9, and details about the obtained DRG with $d \geq 3$ are given in Table 10 .

Remark 6 To the best of our knowledge the strongly regular graphs with parameters $(28431,3150,621,315)$ and $(28431,2880,324,288)$ are the first known examples of SRGs with these parameters. The graphs $\Gamma_{1}^{3}, \Gamma_{2}^{3}, \Gamma_{3}^{3}, \Gamma_{4}^{3}$ and $\Gamma_{5}^{3}$ are rank 3 graphs. The graph $\Gamma_{1}^{3}$ is $N^{-}(7,3)$ graph, $\Gamma_{2}^{3}$ is $O(7,3)$ graph, the graph $\Gamma_{3}^{3}$ is $N O^{+}(7,3)$ graph, the graph $\Gamma_{4}^{3}$ is $N^{+}(8,3)$ graph, and the graph $\Gamma_{5}^{3}$ is $O^{+}(8,3)$ graph. The orthogonal group $O^{+}(8,3)$ acts primitively on the SRGs $\Gamma_{4}^{3}, \Gamma_{5}^{3}, \Gamma_{6}^{3}$ and $\Gamma_{7}^{3}$. Further, strongly regular graphs $\Gamma_{1}^{3}$ and $\Gamma_{3}^{3}$ can be constructed from symmetric incidence matrices with all-zero diagonal of symmetric block designs with parameters $2-(351,126,45)$ and $2-(378,117,36)$, respectively. Similarly, the strongly regular graph $\Gamma_{2}^{3}$ can be constructed from the symmetric incidence matrix with all-one diagonal of a symmetric block design with parameters 2-(364, 121, 40). The full automorphism groups of symmetric block designs in these cases are the same as the automorphism groups of the corresponding SRGs. We checked imprimitive representations of the group $O(7,3)$ up to the degree 10000, and proved that there are no SRGs in these cases. 
Table 9 SRGs constructed from the group $O(7,3)$

\begin{tabular}{lll}
\hline Graph $\Gamma$ & Parameters & Aut $(\Gamma)$ \\
\hline$\Gamma_{1}^{3}=\Gamma\left(O(7,3), H_{1}^{3}\right)$ & $(351,126,45,45)$ & $O(7,3): Z_{2}$ \\
$\Gamma_{2}^{3}=\Gamma\left(O(7,3), H_{2}^{3}\right)$ & $(364,120,38,40)$ & $O(7,3): Z_{2}$ \\
$\Gamma_{3}^{3}=\Gamma\left(O(7,3), H_{3}^{3}\right)$ & $(378,117,36,36)$ & $O(7,3): Z_{2}$ \\
$\Gamma_{4}^{3}=\Gamma\left(O(7,3), H_{4}^{3}\right)$ & $(1080,351,126,108)$ & $O^{+}(8,3): E_{4}$ \\
$\Gamma_{5}^{3}=\Gamma\left(O(7,3), H_{5}^{3}\right)$ & $(1120,390,146,130)$ & $O^{+}(8,3): D_{8}$ \\
$\Gamma_{6}^{3}=\Gamma\left(O(7,3), H_{6}^{3}\right)$ & $(28431,3150,621,315)$ & $O^{+}(8,3): S_{3}$ \\
$\Gamma_{7}^{3}=\Gamma\left(O(7,3), H_{6}^{3}\right)$ & $(28431,2880,324,288)$ & $O^{+}(8,3): S_{3}$ \\
\hline
\end{tabular}

Table 10 DRG constructed from the group $O(7,3), d \geq 3$

\begin{tabular}{lllll}
\hline Graph $\Gamma$ & Number of vertices & Diameter & Intersection array & $\operatorname{Aut}(\Gamma)$ \\
\hline$\Gamma_{8}^{3}=\Gamma\left(O(7,3), H_{5}^{3}\right)$ & 1120 & 3 & $\{39,36,27 ; 1,4,13\}$ & $O(7,3): Z_{2}$ \\
\hline
\end{tabular}

Remark 7 The graph $\Gamma_{8}^{3}$ is the dual polar graph $B_{3}(3)$ (see [6]). We checked imprimitive representations of the group $O(7,3)$ up to the degree 10000, and proved that there are no DRGs in these cases.

\subsection{SRGs and DRGs from the Tits group $T$}

The Tits group ${ }^{2} F_{4}(2)^{\prime}$ or $T$, named after Jacques Tits, is a finite simple group of order $17971200=2^{11} \cdot 3^{3} \cdot 5^{2} \cdot 13$. Up to conjugation it has 434 subgroups, 8 of which are maximal. It is sometimes considered as the 27 th sporadic group since the Tits group itself is not a group of Lie type even the group ${ }^{2} F_{4}(2)$ (which is not simple group) is a group of Lie type. The Tits group $T$ has 10 conjugacy classes of subgroups up to the index 7000. In Table 11 we give the list of all the subgroups $H_{i}^{4} \leq T$ which lead to the construction of SRGs or DRGs of diameter $d \geq 3$.

Using the method described in Theorem 1, we obtained all DRGs with at most 12000 vertices on which the group $T$ acts primitively, and all DRGs with at most 7000 vertices on which the group $T$ acts imprimitively.

Table 11 Subgroups of the group $T$

\begin{tabular}{llllll}
\hline Subgroup & Structure & Order & Index & Rank & Primitive \\
\hline$H_{1}^{4}$ & $L_{3}(3): 2$ & 11232 & 1600 & 4 & Yes \\
$H_{2}^{4}$ & $2.2^{8}: 5: 4$ & 10240 & 1755 & 5 & Yes \\
$H_{3}^{4}$ & $2.2^{8}: S_{3}$ & 6144 & 2925 & 6 & Yes \\
$H_{4}^{4}$ & $2^{5}:\left(\left(2^{4}: 5\right): 2\right)$ & 5120 & 3510 & 10 & No \\
\hline
\end{tabular}


Table 12 SRGs constructed from the group $T$

\begin{tabular}{lll}
\hline Graph $\Gamma$ & Parameters & $\operatorname{Aut}(\Gamma)$ \\
\hline$\Gamma_{1}^{4}=\Gamma\left(T, H_{1}^{4}\right)$ & $(1600,351,94,72)$ & $T$ \\
$\Gamma_{2}^{4}=\Gamma\left(T, H_{4}^{4}\right)$ & $(3510,693,180,126)$ & $F i_{22} .2$ \\
\hline
\end{tabular}

Table 13 DRGs constructed from the group $T, d \geq 3$

\begin{tabular}{lllll}
\hline Graph $\Gamma$ & Number of vertices & Diameter & Intersection array & $\operatorname{Aut}(\Gamma)$ \\
\hline$\Gamma_{3}^{4}=\Gamma\left(T, H_{2}^{4}\right)$ & 1755 & 4 & $\{10,8,8,8 ; 1,1,1,5\}$ & $T: 2$ \\
$\Gamma_{4}^{4}=\Gamma\left(T, H_{3}^{4}\right)$ & 2925 & 4 & $\{12,8,8,8 ; 1,1,1,3\}$ & $T: 2$ \\
\hline
\end{tabular}

Theorem 5 Up to isomorphism there is exactly one SRG and there are exactly two $D R G s$ of diameter $d \geq 3$ with at most 12000 vertices, admitting a primitive action of the group $T$ and exactly one SRG with at most 7000 vertices, admitting an imprimitive action of the group T. The SRGs have parameters $(1600,351,94,72)$ and $(3510,693,180,126)$, and DRGs have 1755 or 2925 vertices. Details about the obtained strongly regular graphs are given in Table 12, and details about the obtained DRGs with $d \geq 3$ are in Table 13 .

Remark 8 The SRGs with parameters $(1600,351,94,72)$ and $(3510,693,180,126)$ are described in [21]. The graph $\Gamma_{2}^{4}$ can be constructed from the group $F i_{22}$ as a rank 3 graph. Moreover, it belongs to the Fisher tower (see [16,24]).

Remark 9 The graph $\Gamma_{3}^{4}$ is the generalized octagon of order $(2,4)$, and the graph $\Gamma_{4}^{4}$ is the generalized octagon of order $(4,2)$. For more information about graph $\Gamma_{3}^{4}$ we refer the reader to [1]. We checked imprimitive representations of the group $T$ up to the degree 7000, and proved that there are no DRGs in these cases.

Remark 10 All SRGs and DRGs constructed in this paper are vertex-transitive, but some of them are also edge-transitive. The graphs that are not edge-transitive are $\Gamma_{2}^{1}$, $\Gamma_{8}^{1}$ and $\Gamma_{1}^{2}$.

Acknowledgements This work has been fully supported by Croatian Science Foundation under the Project 6732. The authors would like to thank Ferdinand Ihringer, William Kantor and Dmitrii Pasechnik for their valuable suggestions. The authors would like to thank the anonymous referees for helpful comments that improved the presentation of the paper.

\section{Appendix}

We give here a construction of new examples of SRGs with parameters (28431, 3150, $621,315)$ and $(28431,2880,324,288)$ using GAP $[15,22,29]$. To simplify the description we give a construction of the SRGs from their automorphism group $O^{+}(8,3)$.

LoadPackage ("atlasrep") ; ; 


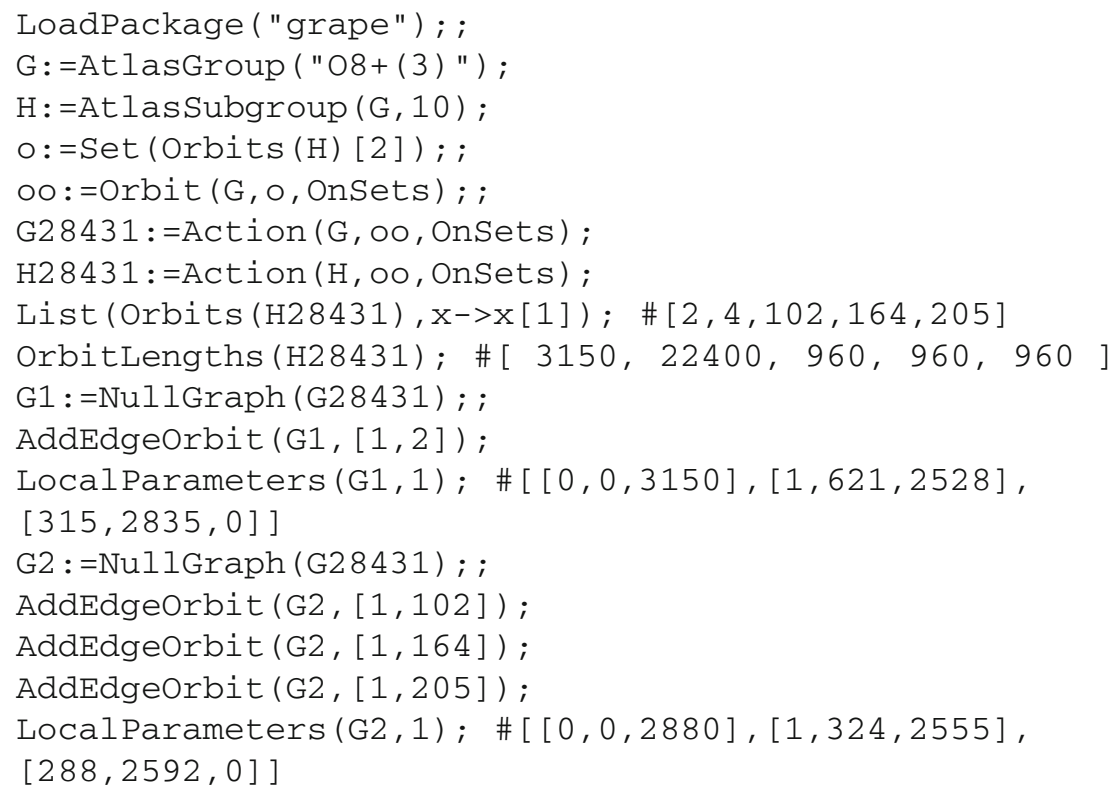

\section{References}

1. Belousov, I.N., Makhnev, A.A.: On automorphisms of generalized octagon of order (2, 4). Mat. Zametki 84, 516-526 (2008)

2. Bose, R.C., Shimamoto, T.: Classification and analysis of partially balanced incomplete block designs with two associate classes. J. Amer. Statist. Assoc. 47, 151-184 (1952)

3. Bosma, W., Cannon, J.: Handbook of Magma Functions. University of Sydney, Department of Mathematics (1994). http://magma.maths.usyd.edu.au/magma

4. Brouwer, A.E.: Strongly regular graphs. In: Colbourn, C.J., Dinitz, J.H. (eds.) Handbook of Combinatorial Designs, 2nd edn, pp. 852-868. Chapman \& Hall/CRC, Boca Raton (2007)

5. Brouwer, A.E.: Parameters of Strongly Regular Graphs. http://www.win.tue.nl/ aeb/graphs/srg/srgtab. html. Accessed 8 Aug 2018

6. Brouwer, A.E., Cohen, A.M., Neumaier, A.: Distance-Regular Graphs. Springer, Berlin (1989)

7. Cameron, P.J.: Coherent Configurations, Association Schemes and Permutation Groups, Groups, Combinatorics \& Geometry (Durham, 2001), 55-71. World Sci. Publ, River Edge (2003)

8. Cohen, N., Pasechnik, D.V.: Implementing Brouwer's database of strongly regular graphs. Des. Codes Cryptogr. 84, 223-235 (2017)

9. Conway, J.H., Curtis, R.T., Norton, S.P., Parker, R.A., Wilson, R.A.: Atlas of Finite Groups. Oxford University Press, Eynsham (1985)

10. Crnković, D., Mikulić Crnković, V., Švob, A.: On some transitive combinatorial structures constructed from the unitary group $U(3,3)$. J. Statist. Plann. Inference 144, 19-40 (2014)

11. Crnković, D., Rukavina, S., Švob, A.: New strongly regular graphs from orthogonal groups $O^{+}(6,2)$ and $O^{-}(6,2)$. Discrete Math. 341, 2723-2728 (2018)

12. van Dam, E.R., Haemers, W.H.: Spectral characterizations of some distance-regular graphs. J. Algebraic Combin. 15, 189-202 (2002)

13. van Dam, E.R., Koolen, J.H., Tanaka, H.: Distance-regular graphs. Electron. J. Combin. (2016), DS22, Dynamic Survey, $156 \mathrm{pp}$

14. Dempwolff, U., Kantor, W.M.: Symmetric designs from the $G_{2}(q)$ heneralized hexagons. J. Combin. Theory Ser. A 98, 410-415 (2002)

15. The GAP Group, GAP_Groups, Algorithms, and Programming, Version 4.8 .7 (2017). http://www. gap-system.org 
16. Hubaut, X.: Strongly regular graphs. Discrete Math. 13, 357-381 (1975)

17. van Lint, J.H., Brouwer, A.E.: Strongly regular graphs and partial geometries. In: Jackson, D.H., Vanstone, A. (eds.) Enumeration and Design, pp. 85-122. Academic Press, London (1984)

18. Pasechnik, D.: Geometric characterization of graphs from the Suzuki chain. European J. Combin. 14, 491-499 (1993)

19. Praeger, C.E., Soicher, L.H.: Low Rank Representations and Graphs for Sporadic Groups. Australian Mathematical Society Lecture Series 8. Cambridge University Press, Cambridge (1997)

20. Robinson, D.: A Course in the Theory of Groups. Springer, New York (1996)

21. Saouter, Y.: Linear binary codes arising from finite groups. In: 2010 6th International Symposium on Turbo Codes \& Iterative Information Processing (Brest, 2010), pp. 83-87. IEEE (2010)

22. Soicher, L.H.: The GRAPE package for GAP, Version 4.7, 2017. http://www.maths.qmul.ac.uk/ $\sim$ leonard/grape/

23. Stein, W.A. et al.: Sage Mathematics Software (Version 7.3). The Sage Development Team (2016). http://www.sagemath.org

24. Tits, J.: Groupes finis simples sporadiques. Sem. Bourbaki 375 (1970)

25. Tonchev, V.D.: Combinatorial Configurations: Designs, Codes, Graphs. Willey, New York (1988)

26. Van Maldeghem, H.: Generalized Polygons. Birkhäuser, Basel (1998)

27. Wielandt, H.: Finite Permutation Groups. Academic Press, New York (1964)

28. Wilson, R.A.: The Finite Simple Groups. Springer, London (2009)

29. Wilson, R.A., Parker, R.A., Nickerson, S., Bray, J.N., Breuer, T.: The GAP package AtlasRep, Version 1.5.1 (2016). https://www.gap-system.org/Packages/atlasrep.html

Publisher's Note Springer Nature remains neutral with regard to jurisdictional claims in published maps and institutional affiliations. 